\title{
Planeación y estrategia: el caso de Productos Alimenticios E1 Pilón
}

Fecha de recepción: 8 de marzo de 2010

Fecha de aprobación: 1 junio de 2010

\author{
Alejandro Castaño Ramírez \\ acastano@poligran.edu.co \\ Politécnico Grancolombiano
}

\section{Reseña del autor}

Docente investigador del programa de Mercadeo y Publicidad del Politécnico Grancolombiano. Antropólogo de profesión y experto en investigación de mercados, con más seis años de experiencia en diseño y dirección de investigaciones aplicadas para agencias de investigación y empresas y entidades académicas. Su perspectiva de la cultura ofrece una mirada nueva al tema del mercadeo estratégico, pues expone la realidad de las pequeñas empresas en Bogotá.

\section{Resumen}

Tradicionalmente se ha sustentado que la actividad de gestión organizacional, o planeación estratégica, no es propia del modelo PYME -pequeña y mediana empresa-, debido a las limitaciones que éste impone, dado su carácter formal y rígido. Pero la influencia de las escuelas de administración en la disciplina del mercadeo ha permitido que los emprendedores usen estos conceptos estratégicos de manera más frecuente. El caso de El Pilón, una microempresa que produce arepas precocidas en Bogotá, Colombia, sirve de ejemplo para la caracterización de la estrategia comercial y el desarrollo de procesos de planeación estratégica.

\section{Palabras clave}

Planeación, estrategia, pequeños negocios, Latinoamérica.

\begin{abstract}
Traditionally, it has been supported that the organizational activity of organizational management, or strategic planning, does not belong to the SME'S organizational model. This is due to the formal and rigid character imposing the companies' commercial activities. But the influence of Business Administration on Marketing is fostering its use among executives and entrepreneurs. The case of El Pilón, a SME that produce pre-cooked cornbread in Bogotá, Colombia, exemplifies the way a commercial strategy is set and then performed the strategic planning procedure.
\end{abstract}

\section{Key words}

Planning, Strategy, SME'S, Latin-America. 


\section{Introducción}

El pilón es un artefacto utilizado frecuentemente por los indígenas en todo el continente americano para la transformación del grano seco de maíz en harina. A partir de ésta se elaboran alimentos como las tortillas y las arepas.

La fabricación de las arepas, por lo tanto, ha tenido un contexto masivo pero individualizado, en el que cada unidad social produce sus propias arepas. Sin embargo, el mercado de arepas en Colombia muestra una tendencia de crecimiento e industrialización desde hace varios años.

El motivo de este artículo no es el análisis de los cambios culturales que han motivado la compra de las arepas sobre su fabricación en casa, pero esto es relevante puesto que en dichas condiciones surge Productos Alimenticios El Pilón.

El contexto comercial de mercado de arepas precocidas se da en tres canales: el "tradicional" -en tiendas de barrio-, el de supermercados o grandes superficies y el institucional. La experiencia comercial de estrategia y planeación que se estudia en este artículo, se mueve en los primeros dos canales.

Las diferencias entre el canal tradicional y el de supermercados corresponden a las diferencias en los mercados y marcan condiciones para la formulación de estrategias comerciales. El primero está relacionado con la alta informalidad y los bajos niveles de regulación de calidad, mientras que el segundo se asocia con características esencialmente opuestas (Lozano, 2008, p. 3).

El caso de la marca de arepas precocidas El Pilón muestra cómo una PYME enfrenta comercialmente estos dos mercados de competencia en Bogotá, a partir del diseño de estrategias para competir y la generación procesos de gestión, como la planeación estratégica.

Este artículo es resultado de una investigación empírica, basada en un procedimiento metodológico de caso, mediante herramientas cualitativas de investigación. La información generada en este proceso no sólo incrementa el conocimiento académico de los pequeños negocios, sino que brinda herramientas de consideración para todos los involucrados en el desarrollo socioeconómico, tanto entidades gubernamentales como no gubernamentales; también es útil para quienes desarrollan programas de formación de habilidades empresariales para emprendedores, como las cámaras de comercio y consultoras empresariales.

\section{Las PYMEs y los conceptos de planeación y estrategia}

Con frecuencia se argumenta que los pequeños negocios tienen deficiencias en aspectos administrativos (Calle y Tamayo, 2005, p. 155), entre las que se cuenta la carencia de procesos efectivos 
de planeación estratégica (Lawrence, 2007, p. 78). Pero la literatura también da voz a otras opiniones, incluso contrarias a ésta; así, desde hace más de treinta años, las investigaciones muestran que los procesos de gestión sobre planeación estratégica se adoptan en la medida en que una empresa crece y el alcance de gestión del emprendedor se ve reducido a un porcentaje de las operaciones de la empresa (Mintzberg \& Waters, 1982, p. 489; Masurel \& Smit, 2000, p. 96).

Además, las tradiciones académicas de administración y su profusión en forma de cursos y capacitaciones a través de revistas y periódicos, han involucrado la necesidad de procesos como la planeación estratégica para el éxito comercial (Sanabria, 2006, p. 62), y hoy en día algunas posturas y escuelas las consideran indispensables (Brooksbank, 1996, p. 19).

La literatura sobre el tema considera que es inapropiado aplicar conceptos asociados a la gestión de grandes empresas -como la planeación estratégica- a las pymes, (Jocumsen, 2004, p. 622; Culkin \& Smith, 2000, p. 148). Para estos autores, las diferencias estriban en la naturaleza de los procesos de escogencia de una estrategia comercial, que dependen del tamaño de las organizaciones.

La primera diferencia es que las grandes empresas adoptan procesos de planeación de estrategias comerciales explícitos y racionales (Cassar \& Gibson, 2007, p. 301), cuya función es "alinear las acciones con las metas" (Jordan 2003, p. 45). Dichos procesos se consideran inadecuados para los negocios pequeños porque la estrategia comercial de este tipo de organizaciones es manejada de una manera flexible y con alta adaptabilidad a los cambios del ambiente comercial (Culkin \& Smith, 2000, p. 200).

La segunda diferencia es que, en las pequeñas y medianas empresas, la estrategia se formula de una manera implícita (Hill, Nancarrow \& Right, 2002, p. 363), guiada por la emocionalidad (Jocumsen, 2004, p. 624) y altamente condicionada por la personalidad del empresario (Rauch, Frese \& Sonnentag, 2000, p. 37; Miles \& Snow 1978, p. 54), mientras que en las grandes empresas sucede lo contrario.

Aparte de estas diferencias, investigaciones académicas muestran evidencias que indican que la elaboración de procesos de planeación estratégica no es una condición necesaria para el éxito empresarial (Mulhare, 1999, p. 324). Algunos autores consideran que estos procesos son inflexibles y dificultan la innovación y creatividad (Jocumsen, 2004, p. 627; Jordan, 2003, p. 46; Jocumsen 2004, p. 627). Es preciso aclarar que la aplicación de estos procesos no es necesaria en todos los contextos, puesto que la implementación de la planeación estratégica y el éxito comercial están condicionados por dimensiones culturales y geográficas (Rauch et al., 2000, p. 40). 
Estos postulados tienen un origen común en lo propuesto por Geert Hosftede acerca de los efectos de la cultura sobre el desempeño económico (1981, p. 125). Para este autor, la evasión de la incertidumbre es una dimensión cultural que muestra una tendencia social a enfrentar el futuro de una manera regulada, a través de leyes y normas, además de procesos de gestión como la planeación estratégica (Hofstede \& Hofstede 2005, p. 86). Este panorama académico permite la identificación de elementos teóricos con relación a la planeación estratégica en pequeños negocios, que se debe probar con datos primarios y explorar con metodologías cualitativas.

En resumen, los elementos que competen a la planeación estratégica son: la implementación de procedimientos de planeación estratégica en pequeños negocios de acuerdo a su ciclo de vida, la formación explícita e implícita de dichos procesos, la importancia de la personalidad del emprendedor, la tendencia emocional en la toma de decisiones comerciales y la correlación entre evasión de la incertidumbre y planeación estratégica.

\section{Metodología}

Con el fin de explorar cómo se desarrollan estrategias comerciales y cómo se utiliza el concepto de planeación estratégica, se llevó a cabo un estudio longitudinal, que consiste en el seguimiento a un sujeto de estudio durante una temporada; es decir, un estudio de caso, que permite la interacción a profundidad y la verbalización de procesos subconscientes (Denzin \& Linconl 2005, p. 135). Dicha decisión se tomó teniendo en cuenta que las estrategias no cambian día a día, sino que se forman en el tiempo y cambian a lo largo del mismo (Mintzberg, 1981, p. 323).

Se utilizó un instrumento semiestructurado que garantizó que, en las repetidas interacciones, fuera posible abordar diferentes temas, y pudiera aplicarse con diferentes personas (Bonilla-Castro y Rodríguez, 1997, p. 76).

A través del estudio de caso se reconstruyó la historia empresarial y, a lo largo de ésta, se identificaron los procesos de formación de planeación estratégica.

\section{Las estrategias y la planeación en El Pilón}

Productos Alimenticios El Pilón es una empresa que tiene diez años de antigüedad en el mercado. Actualmente, cuenta con quince empleados y reporta ingresos de sesenta a setenta millones de pesos mensuales. Es dirigida por una mujer de treinta y seis años, junto con su esposo, asesor financiero y socio capitalista. La empresa está ubicada en el barrio Rionegro, en el noroccidente de Bogotá. 
Ellos compraron la marca Arepas Santa Rosa, especializada en la fabricación de arepas paisas, a una pareja que quería retirarse del mundo comercial. La mujer de la pareja se retiró de su trabajo y se dedicó a la empresa, en tanto que él conservó su trabajo en una multinacional, también estaba vinculado profesional y financieramente con la empresa.

Tras la adquisición de la marca, sus propietarios hicieron un análisis de mercado, mediante el cual identificaron que la producción de las arepas precocidas que se encontraban en las tiendas se caracterizaban por su bajo nivel de tecnificación, y a la comercialización en zonas específicas de la ciudad.

Entonces surge una estrategia: darle personalidad tecnificada y segura a la marca, en otras palabras, institucionalizarla. Así que los socios invirtieron en el diseño de un logo que la identificara, en maquinaria para empacado por sellado y en la certificación requerida por el INVIMA -Instituto Nacional de Vigilancia de Medicamentos y Alimentos-, para la comercialización de alimentos en Colombia. La estrategia logró el resultado esperado: la marca obtuvo un valor diferencial al introducir en el mercado un producto mejor presentado que el de la competencia y, en consecuencia, las ventas crecieron.

Obtener la certificación no fue fácil por el desconocimiento de la empresaria acerca el tema y la intransigencia del INVIMA, cuyos funcionaros la sometieron a evaluaciones sin una capacitación previa. Esto último conduce a pensar en la imagen del Gobierno como una interpretación sociocultural de las fuerzas que regulan los mercados, de modo que se convierte en un agente que parece estar en contra del desarrollo económico de las microempresas.

Luego de múltiples conflictos para la obtención de la certificación, la marca logró su registro y completó su proceso de institucionalización. Las ventas se han incrementado, las inversiones en presentación y registro son valoradas por el cliente y se gana participación en el mercado a través de una estrategia basada en diferenciación por calidad.

En cuanto a los precios, se adoptó otra estrategia que consistió en amortiguar los costos de los procesos de institucionalización a largo plazo, incrementando de manera mínima los precios al cliente -cien o doscientos pesos-, debido a que los tenderos se negaban a comprar productos por encima de un valor determinado, no obstante las diferencias percibidas en calidad.

Dos años después, tras controlar la distribución en la zona de Rionegro mediante el canal tradicional, los propietarios decidieron ofrecer su producto en grandes almacenes, sin descuidar los mercados ya ganados.

La entrada al mercado de las grandes superficies se inició con la compra de La Bumanguesa, otro pequeño negocio de producción de arepas, especializado en arepas santandereanas. 
La pareja de emprendedores compró las fórmulas, algunos equipos técnicos y la marca, que estaba registrada como proveedora de Surtifruver, una cadena de supermercados especializados en el comercio de frutas y verduras. Al incursionar en este mercado, comenzó la competencia con la marca El Pilón, que compraron posteriormente y le da el nombre a la razón social actual. Al adquirir esta marca, accedieron al hipermercado Carrefour, y así continuaron con la estrategia de entrada a supermercados.

En ese momento, la emprendedora evaluó el comportamiento comercial de su competencia, y encontró algunas debilidades en las estrategias de mercadeo. En consecuencia, formuló una estrategia basada en la competencia en el punto de venta. Contrató impulsadoras, dio degustaciones e hizo seguimiento cercano al comportamiento de su marca en el supermercado. En ese momento propone y desarrolla el Festival de la Arepa, evento que incluía descuentos, promociones y degustaciones. La idea fue tan exitosa que se institucionalizó en Surtifruver, y además la marca ganó la confianza y apoyo del personal del supermercado.

Pero el crecimiento alcanzado hasta el momento fue interrumpido. Pese al incremento inicial de las ventas, gracias a la implementación de la estrategia descrita anteriormente, el hipermercado le hizo muchas devoluciones, que aumentaron progresivamente. La emprendedora realizó un seguimiento, y encontró que debía tener mercaderistas e impulsadoras en cada hipermercado para garantizar la exhibición y rotación del producto. Lo hizo, pero aún así la situación no cambió. Este fue uno de los momentos más difíciles de la empresa. Las ventas bajaron y además, por enfocar su atención en las grandes superficies, descuidaron los mercados ya colonizados.

Pocos meses después decidieron retirar sus productos de los hipermercados, pues consideraban que continuar allí significaba la bancarrota. Así, abandonaron la estrategia comercial diseñada para las grandes superficies y se centraron en reconquistar los mercados que una vez tuvieron. De este modo, la empresa recuperó su punto de equilibrio.

A partir de esta experiencia, la pareja de emprendedores diseña y dirige la planeación estratégica de esta empresa. Cada año, reúnen información interna y de comportamiento de los mercados para plantearse objetivos y metas, y este procedimiento queda escrito en documento.

\section{Discusión}

A pesar de los problemas que enfrentó Productos Alimenticios El Pilón, no es posible relacionarlos directamente con carencias en conceptos administrativos de la estrategia, como sostienen algunas investigaciones revisadas al inicio de este 
artículo, ya que el proceso de planeación es llevado a cabo de una manera formal, como lo expone la teoría.

La presencia de este comportamiento es reflejo de la formación comercial de la pareja de empresarios, quienes cuentan con antecedentes en la toma estructurada de decisiones comerciales, así como formación académica con tendencias administrativas. Por otro lado, se corrobora el impacto de la personalidad el emprendedor sobre las determinaciones estratégicas, como lo asevera la literatura.

El fenómeno de formalización de la estrategia en esta pequeña empresa muestra cómo las características formales no son rígidas, ni disminuyen la capacidad de acción de la empresa, a pesar de su tamaño. Antes bien, puede considerarse como un ejercicio susceptible de ser abandonado, tras una reflexión previa. Esto deja de lado la idea sostenida en la literatura de que el proceso de planeación conlleva a la rigidez. Claro, esto debe observarse desde el contexto cultural, puede que opere de manera diferente en otra región de la ciudad u otra actividad económica.

La evasión de la incertidumbre es una dimensión por medir, pero en Productos Alimenticios El Pilón se ha encontrado de manera cualitativa que la incertidumbre y percepción de volatilidad de los mercados está presente todo el tiempo.

La planeación estratégica se presenta como un ritual que permite conjurar las fuerzas desconocidas del futuro y establecer objetivos realizables. Aún así, su fuerza parece débil y las estrategias de la empresa deben ajustarse a lo largo del proceso, en un plazo de un año -lo que considera una visión de corto plazo-.

Por último, encontramos que la literatura indica que la planeación en las PYMEs tiende a ser altamente emocional, se basa en la información más preponderante en la sociedad, que no siempre es correcta, y esto sucede en el caso de Productos Alimenticios El Pilón. Cuando se plantea la entrada a los supermercados como estrategia comercial, se toma la decisión basado en el imaginario cultural que considera ese canal como el punto más alto de la cadena de distribución; pero ellos no buscaron o compararon esta posibilidad con otras experiencias para desempeñarse mejor, sino que se guiaron por la emoción de llegar al supuesto punto más alto de la cadena.

\section{Conclusiones}

La reconstrucción de las estrategias utilizadas por El Pilón y de la manera como se plantean, muestra cómo la empresa y su pareja de emprendedores tienen una personalidad formada a partir de la práctica del mercadeo y la administración en compañías multinacionales.

La escritura de una planeación estratégica es resultado de esta formación, pero el desarrollo de ésta es bastante 
amplio, pues les permite abandonar lo planeado, frente a las circunstancias de mercados desconocidos. La emprendedora considera que la mayor cantidad de errores se cometen a causa del desconocimiento de los mercados, como le sucedió con los hipermercados, y de los requisitos comerciales, con el INvima.

Se observa un componente altamente emocional, evidenciado en el deseo de entrar a grandes superficies, a pesar del desconocimiento del mercado y los riesgos que efectivamente trajo el comportamiento emocional a la empresa. Esto se relaciona ampliamente con la tendencia intuitiva en los negocios que se le atribuye a las pymes, puesto que demuestra la falta de búsquedas de información adecuadas que ayuden a perfilar un negocio con éxito.

De igual manera, se detectó la presencia del ritual de la planeación como un evento en el que, una vez al año, se conjuran las fuerzas del futuro a través del análisis de la organización, y se planea para controlar el caos.

\section{Referencias}

1. Bonilla-Castro, E. y RodríGuez, P. (1997). Más allá del dilema de los métodos. (3ra Ed.). Bogotá: Grupo Editorial Norma, Universidad de los Andes.

2. Brooksbank, R. (1996). The Basic Marketing Planning Process: a
Practical Framework for the Smaller Business. Marketing Intelligence Ẻ Planning, Núm.14 (4), pp. 16-22.

3. Calle, A.y Tamayo, V. (2005). Estrategia e internacionalización en las Pymes: caso Antioquia. Cuadernos de Administración, Núm. 18 (30), pp. 137-164.

4. Cassar, G. \& Gibson, B. (2007). Forecast Rationality in Small Firms. Journal of Small Business Management, No. 45 (3), pp. 283-302.

5. Culkin, N. \& Smith, D. (2000). An Emotional Business: a Guide to Understanding the Motivations of Small Business Decision Takers. Qualitative market research, No. 3 (3), pp. 145.

6. Denzin, N. \& Lincoln, Y. (2005). Handbook of qualitative research. California: Sage Publications.

7. Hill, J.; Nancarrow, C. \& Right, L. (2002). Lifecycles and Crisis Points in smes: a Case Approach. Marketing Intelligence and Planning, No. 20 (6), pp. 361-369.

8. Hofstede, G. \& Hosftede, G.J. (2005). Cultures and Organizations. New York: McGraw-Hill.

10. Jocumsen, G. (2004). How do Small Business Managers Make Strategic Marketing Decisions? European Journal of Marketing, No. 38 (5/6), pp. 659-674.

11. Jordan. A.T. (2003). Business Anthropology. Long Grove, Illinois: Waveland Press Inc. 
12. Gibbons, P.T. \& O‘Connor, T. (2005). Influences on Strategic Planning Processes among Irish smes. Journal of Small Business Management, No. 43 (2), pp.170-186.

13. Lawrence, W. (2007). Small Business Operations Strategy: Aligning Priorities and Resources. Journal of Small Business Strategy, No. 19 (2), pp. 89-103.

14. Lozano, R (2009, marzo 19). Mercado de arepas precocidas alcanzó en Colombia $\$ 3,9$ billones, con un crecimiento de 3\% en 2008. Portafolio. Recuperado el 23 de julio de 2009 desde http://www.portafolio.com. co/negocios/empresas/2009-03-18/ ARTICULO-WEB-NOTA_INTERIOR_PORTA-4883106.html

15. Masurel, E. \& Smit, H. (2000). Planning Behavior in Small Firms in Central Vietnam. Journal of Small Business Management, No. 38 (2), pp. 95-102.
16. Mulhare,E.(1999).Mindful of the Future: Strategic Planning Ideology and the non-Profit Management. Human Organization, No. 58 (3), pp. 323-330.

17. Miles, R.E. \& Snow, C.C. (1978). Organization, Strategy, Structure, Process. New York: McGraw-Hill.

18. Mintzberg,H.;Waters,J.(1982). Tracking Strategy in an Entrepreneurial Firm. Academy of Management Journal, No. 25, pp. 465-499.

19. Rauch, A.; Frese, M. \& SonnenTAG, S. (2000). Cultural Differences in Planning/Success Relationships: a Comparison of Small Enterprises in Ireland, West Germany and East Germany. Journal of Small Business Management, No. 38 (4), pp. $28-41$.

20. Sanabria, M. (2004). El pensamiento organizacional estratégico: una perspectiva diacrónica. Innovar: revista de ciencias administrativas y sociales, Núm. 24, pp. 59-81. 